\title{
Product Eco-Design in the Era of Circular Economy: Experiences in the Design of Espresso Coffee Machines
}

\author{
Claudio Favi ${ }^{1}$, Marco Marconi $^{2(凶)}$, Marta Rossi $^{3}$, and Federica Cappelletti ${ }^{3}$ \\ ${ }^{1}$ Department of Engineering and Architecture, Università degli Studi di Parma, Parco Area \\ delle Scienze 181/A, 43124 Parma, Italy \\ 2 Università degli Studi della Tuscia, Largo dell'Università, 01100 Viterbo, Italy \\ marco.marconi@unitus.it \\ 3 Department of Industrial Engineering and Mathematical Sciences, Università Politecnica delle \\ Marche, Via Brecce Bianche, 60131 Ancona, Italy
}

\begin{abstract}
Product eco-design includes several methodologies aimed at supporting companies in the development of sustainable products. Currently, this theme is assuming an important role in both the academia and industry worlds due to the increasing attention to environmental problems and the need for a transition toward circular economy business/organizational models. In this context, the present paper focuses on the industrial sector of espresso coffee machines manufacturing which has several unexploited potentialities. The analysis of the sector specificity (internal and external contexts), as well as of the product lifecycle allowed to define an eco-design framework to guide companies involved in the design and production of espresso coffee machines. Effective eco-design strategies should include the combined use of specific methods, tools and metrics to manage all the most important lifecycle phases (beginning of life, middle of life, end of life) during the design activities in order to set preventive actions that avoid future potential environmental impacts. Only in this way, the environmental and economic benefits of the circular economy paradigm (e.g. remanufacturing/reuse of selected components) can be practically exploited in real industrial contexts. The presented case studies confirmed that the application of design for disassembly rules positively contributes to increase the product performances during maintenance and end of life, while a re-design oriented to component modularity could be a key strategy to pursue remanufacturing for boilers, a key and expensive component included in espresso coffee machines.
\end{abstract}

Keywords: Product eco-design · Circular economy $\cdot$ Remanufacturing · Recycling · Coffee machine

\section{Introduction}

Eco-design includes a set of design methodologies that support technical departments in the development of sustainable products. Several key factors are forcing companies 
to launch in the market products with a minimized environmental load: new legislations, the opportunity to enlarge the market share, economic advantages, brand preservation, increasing environmental awareness of customers [1]. This context stimulated the development of hundreds of general-purpose or sectorial eco-design methods and tools from both the scientific and industrial world [2]. In recent years, the concept of circular economy (CE) acquired importance as a business/organizational model focused on the increasing of product lifetime, minimization of scraps/wastes, implementation of closed-loop end of life (EoL) scenarios [3]. Currently, the implementation of CE models is mainly based on "remedial" actions that foresee the recovery of products and materials discarded at the end of their useful life. Too many decisions are still made without a strategy, resulting in higher costs and lower success rate, while eco-design must be a proactive management approach [4]. However, such approaches have a limited efficiency and only a transition to "preventive" actions can lead to full benefits of the CE paradigm [5]. This is the main reason why in the last years the scientific community is increasingly focused on the proposal of design methodologies aimed at supporting the development of products that are easy to manage and recover at EoL. One of the most widespread is design for disassembly (DfD), a target design methodology focused on the product disassembly, a preliminary but essential phase for both maintenance/repair of products during the use phase, and at the EoL, in order to favor the component reuse/remanufacturing and the material separation/recycling [6]. Even if disassembly occurs during the lifetime or at the EoL, the choices made at the design phases strongly influence the disassembly performance. For this reason, several literature studies are focused on identifying the optimized disassembly sequence for components with high residual value [7], defining calculation methods and new disassemblability metrics (e.g. time, cost), evaluating EoL performance, developing tools for supporting design activities [8].

The present paper aims to investigate how existing eco-design methods, tools and metrics can be used in the context of espresso coffee machines. The characterization of such industrial sector and products provided several eco-design actions that can be reinjected in other field and in the development of other products. The novelty consists in defining a framework to guide companies belonging to this sector in the transition toward eco-sustainability and the exploitation of CE opportunities, through the implementation of eco-design methods and tools.

After the Introduction that clarifies the general context, Sect. 2 explains how ecodesign can be applied in case of coffee machine production, detailing the most useful and suitable methods, tools and metrics. Section 3 presents some experiences of eco-design implementation carried out in collaboration with an Italian company. Finally, Sect. 4 discusses outcomes, limitations and future work.

\section{Product Eco-Design for Coffee Machines}

Starting from the analysis of eco-design methods and tools, a customization for professional coffee-machine sector is proposed; a selection of tools is identified, and benefits related to their implementation are discussed considering the life cycle phases and the issues faced.

A professional espresso coffee machine is a complex product whose main functionality is to support the barman in the preparation of coffee-based drinks. Analyzing the 
product and the different lifecycle phases, it is clear that to effectively pursue eco-design, several internal and external factors need be taken into account. Specific legislations (e.g. EU directives on eco-design or waste of electric equipment), customer requirements (e.g. high-end vs low-end markets, customer awareness on environmental themes), company business objectives, the complexity of certain key components/functional groups (e.g. boiler, brewing groups), the high consumption of energy during the long lifetime (up to 10-15 years), the difficulty to manage the product at EoL and exploit CE potentialities, are some of the aspects to be considered, forcing to take into account the whole product lifecycle. Different eco-design strategies and metrics need to be simultaneously used to have sensible benefits and avoid burden shifting, as depicted in Fig. 1.

Beginning of Life (BoL) includes all the phases needed for the coffee machine manufacturing until its distribution and use. Due to the complexity of such products a high number of heterogeneous materials are commonly used (e.g. steel for frame, copper for boilers, aluminum for other internal parts), brass or aluminum for brewing groups, different typologies of plastics for internal parts and the external case, stainless steel in case of high-end market products). Moreover, it must be considered that material choice strongly influences the performances of the successive phases (e.g. recyclability or possibility to separate material at EoL). Therefore, eco-design in the context of coffee machine BoL requires the use of material selection tools or at least a material database including environmental properties of materials (e.g. Ansys Granta Selector tool equipped with the Ansys Granta MI database). The use of such tools allows anticipating sustainable matters yet in the first design phase and reduce the environmental impact of products.

Middle of Life (MoL) is a key phase, since it is well known that most of the environmental impacts for energy using products is caused during their use (up to 95-98\% in case of coffee machines). The energy consumption is the most important metric concerning the use phase. Its accurate forecast and/or measurement through standard protocols and use scenarios (not existing at the moment in the field of coffee machines) are essential aspects to implement effective eco-design actions (e.g. thermal insulation of boilers and pipes for heat dissipation reduction). Concerning Life Cycle Assessment (LCA) indicators to be considered for the use phase analysis, the Global Warming Potential (GWP measured in [kgCO2eq]) is certainly the most suitable and useful indicator for coffee machines, as also confirmed by recent literature studies [9].

End of Life includes all the phases successive to the product discard at the end of the useful life. The monitoring of the product/component disassemblability during the design phase is a key activity to guarantee the implementation of closed-loop EoL strategies. DfD metrics, as disassembly time and cost, must be quantified for target components, on the basis of available design data (e.g. 3D models, product structure, bill of materials), by using dedicated methods and tools [8]. In addition, EoL indices should be exploited during the coffee machine design to preliminarily verify the feasibility and convenience of a certain EoL scenario (e.g. remanufacturing), and focus the design for EoL strategy toward the right direction [10]. 


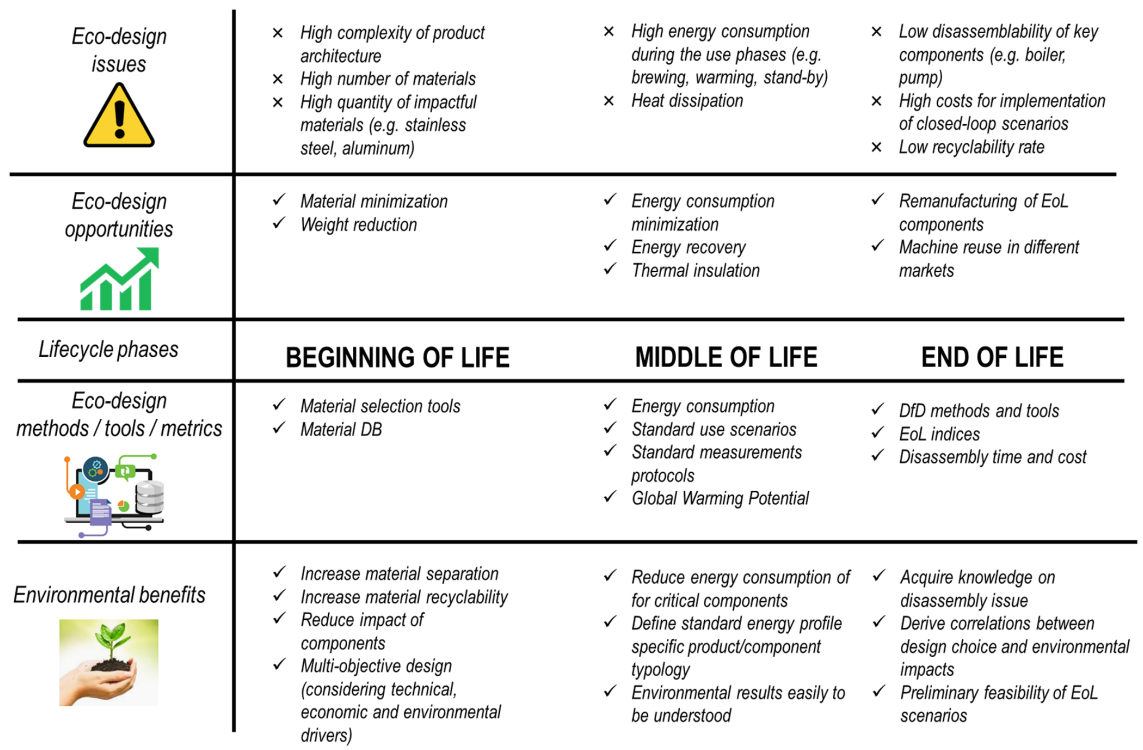

Fig. 1. Correlations among lifecycle phases, eco-design issues/potentialities and most suitable supporting methods/tools/metrics.

\section{Case Studies}

In this section, few examples of good practices oriented to eco-design are presented. Most of them refers to the product EoL that is one of the most critical phases to consider in this century when virgin resources are becoming scarce, and the management of domestic and industrial wastes is a key issue for environment.

The first example proposed within this study is the reduction of disassembly time for target components (Fig. 2). Reduction of disassembly time is directly correlated to the reduction of disassembly operations (i.e. decrease the number of components to disassemble) and the reduction of time for each operation (i.e. faster assembly/disassembly connectors). In Fig. 2 the target component is the "plastic bowl" used to contain water. Bowl requires to be disassembled during the product use for water refilling and because this component is subjected to damage. The redesign action included the integration of different component in a single one (made by only one type of plastic) and the possibility to easy disassemble it with the adoption of cylindrical plastic snap-fits, instead of the original threaded joints.

Connected with the disassembly phase, EoL business models oriented to $\mathrm{CE}$ (i.e. component remanufacturing, product refurbishment) are new important paradigms to pursue for companies that want to increase their business volumes. To this aim, key components with high material and manufacturing costs require a specific design. This is the case of the "main boiler" which requires to be manufactured with copper-based materials (i.e. copper or brass) due to their thermal performances. The design of main boiler changed to adapt different connectors for other espresso machine models (Fig. 3). Indeed, this component can be remanufactured with the aim to be reused in a large number 

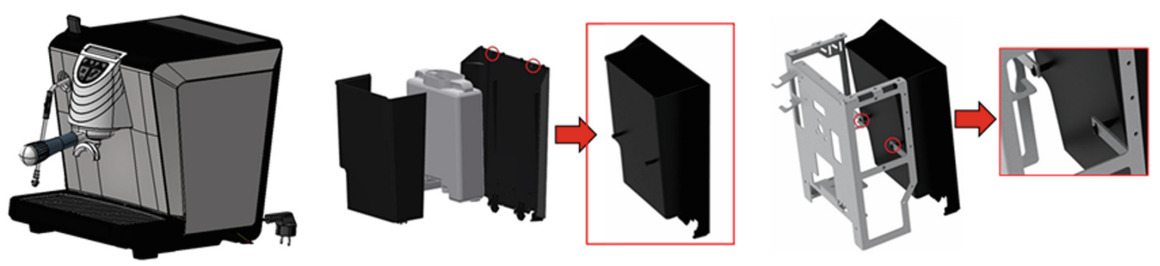

Fig. 2. Examples of design modifications to decrease disassembly time of plastic parts.

of models. The most important design change was related to the flange/cap that was adapted to several configurations and attachments with other components. Environmental analysis should be always coupled with cost analysis. Even remanufacturing option requires additional costs, they are lower than the economic profits provided by the implementation of this new business model.

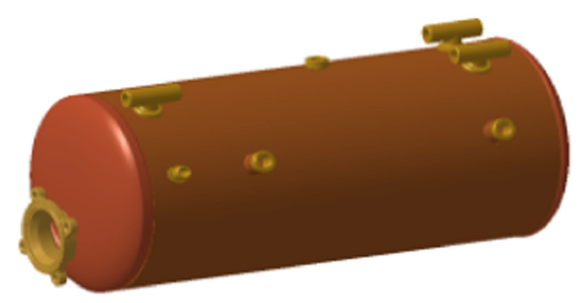

A

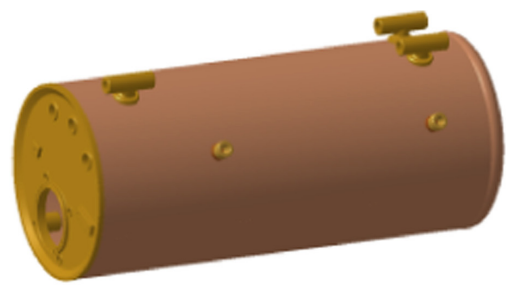

$\mathrm{B}$

Fig. 3. Examples of design modifications for the main boiler (remanufacturing purpose): Original design (A) vs New design (B).

\section{Conclusions}

This paper investigates eco-design methods in the specific context of espresso coffee machine product development. The specificity of this product deals with the adoption of dedicated eco-design actions and metrics to implement CE business models. Few examples were discussed providing some models that can be exploited also in other contexts: (i) disassembly, (ii) recycling, and (iii) remanufacturing. While disassembly is a key performance indicator in the analysis of EoL options for target components, recycling and remanufacturing are new business models that can be implemented after a technical, economic and environmental analyses. Within the case study the re-design of target components allowed to implement emerging business models (i.e. product remanufacturing) encouraging the circular economy paradigm. Future work will be focused on the definition of key performance indicators (EoL indices) dedicated to the espresso coffee machine sector, able to consider the three pillars of sustainability: (i) economic, (ii) environmental, and (iii) social. These indicators can be used as decision-making tool in the assessment of design alternatives as well as in the definition of eco-design actions. 


\section{References}

1. Bey, N., Hauschild, M.Z., McAloone, T.C.: Drivers and barriers for implementation of environmental strategies in manufacturing companies. CIRP Ann. 62(1), 43-46 (2013)

2. Dekoninck, E.A., Domingo, L., O'Hare, J.A., Pigosso, D.C.A., Reyes, T., Troussier, N.: Defining the challenges for ecodesign implementation in companies: development and consolidation of a framework. J. Clean. Prod. 135, 410-425 (2016)

3. Reike, D., Vermeulen, W.J.V., Witjes, S.: The circular economy: new or refurbished as CE 3.0? - exploring controversies in the conceptualization of the circular economy through a focus on history and resource value retention options. Resour. Conserv. Recycl. 135, 246-264 (2018)

4. Pigosso, D., Zanette, E.T., Filho, A.G., Ometto, A.R., Rozenfeld, H.: Ecodesign methods focused on remanufacturing. J. Clean. Prod. 18, 21-31 (2010)

5. Vanegas, P., Peeters, J.R., Cattrysse, D., Tecchio, P., Ardente, F., Mathieux, F., Dewulf, W., Duflou, J.R.: Ease of disassembly of products to support circular economy strategies. Resour. Conserv. Recycl. 135, 323-334 (2018)

6. Duflou, J.R., Seliger, G., Kara, S., Umeda, Y., Ometto, A., Willems, B.: Efficiency and feasibility of product disassembly: a case-based study. CIRP Ann. 57(2), 583-600 (2008)

7. Kara, S., Pornprasitpol, P., Kaebernick, H.: A selective disassembly methodology for end-oflife products. Assem. Autom. 25(2), 124-134 (2005)

8. Favi, C., Marconi, M., Germani, M., Mandolini, M.: A design for disassembly tool oriented to mechatronic product demanufacturing and recycling. Adv. Eng. Inform. 39, 62-79 (2019)

9. Hicks, A.L., Halvorsen, H.: Environmental impact of evolving coffee technologies. Int. J. Life Cycle Assess. 24, 1396-1408 (2019)

10. Favi, C., Germani, M., Luzi, A., Mandolini, M., Marconi, M.: A design for EoL approach and metrics to favour closed-loop scenarios for products. Int. J. Sustain. Eng. 10(3), 136-146 (2019)

Open Access This chapter is licensed under the terms of the Creative Commons Attribution 4.0 International License (http://creativecommons.org/licenses/by/4.0/), which permits use, sharing, adaptation, distribution and reproduction in any medium or format, as long as you give appropriate credit to the original author(s) and the source, provide a link to the Creative Commons license and indicate if changes were made.

The images or other third party material in this chapter are included in the chapter's Creative Commons license, unless indicated otherwise in a credit line to the material. If material is not included in the chapter's Creative Commons license and your intended use is not permitted by statutory regulation or exceeds the permitted use, you will need to obtain permission directly from the copyright holder.

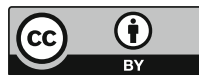

